\title{
XMM-Newton observation of the Seyfert 1 galaxy Fairall 9
}

\author{
P. Gondoin ${ }^{1}$, D. Lumb ${ }^{1}$, H. Siddiqui ${ }^{1}$, M. Guainazzi ${ }^{2}$, and N. Schartel ${ }^{2}$ \\ 1 Astrophysics Division, European Space Agency - Postbus 299, 2200 AG Noordwijk, The Netherlands \\ 2 ESA Villafranca Satellite Tracking Station, Apartado 50727, 28080 Madrid, Spain
}

Received 20 February 2001 / Accepted 19 April 2001

\begin{abstract}
We report an X-ray observation of the Fairall 9 Seyfert 1 galaxy performed in July 2000 with the EPIC cameras and Reflection Grating Spectrometers (RGS) on board the XMM-Newton observatory. Above $2 \mathrm{keV}$, the high signal to noise ratio of the EPIC spectra shows an emission line at $6.38 \pm 0.03 \mathrm{keV}$ with a measured equivalent width of $120 \mathrm{eV}$. An absorption edge is also detected at $7.64_{-0.36}^{+0.21}$ in the source rest frame that has not been reported before. Spectral fitting of the measured spectra with reflection models indicates that these imprinted features are likely the result of partial reprocessing of a primary X-ray continuum by optically thick material. The positions in energy of the emission line and absorption edge are consistent with Compton reflection models from material containing iron in states of ionization lower than FeXVII. The Fe K emission line is narrow suggesting that the reflection process arises from material relatively far from a putative central black hole. The best fit models show that the reflected spectrum mask a steeper intrinsic power law slope even at energies lower than $5 \mathrm{keV}$. The extrapolation of this primary continuum to energies lower than $2 \mathrm{keV}$ reveals the presence of a soft excess component contributing to $14 \pm 6 \%$ of the overall flux in the $0.3-1.0 \mathrm{keV}$ energy range. No evidence is found for a warm absorber in this low energy part of the spectrum. The analysis results are discussed within the frame of recent accretion disc models in which X-rays from the primary continuum source heat up the upper layers of the disc thus creating very hot, optically thin plasmas on top of cooler denser material.
\end{abstract}

Key words. galaxies: individual: Fairall 9 - galaxies: nuclei - galaxies: Seyfert

\section{Introduction}

Fairall 9 is a Seyfert 1 galaxy, at a redshift $z=0.0461$ (Ward \& Wilson 1978). Its morphology in the visible spectral band shows an unresolved nucleus $\left(<1^{\prime \prime}=890 \mathrm{pc}\right.$ at $180 \mathrm{Mpc}$ assuming $H_{0}=75 \mathrm{~km} \mathrm{~s}^{-1} \mathrm{Mpc}^{-1}$ ) with an envelope extending up to $20^{\prime \prime}$ from the center and an elliptical companion galaxy at $28^{\prime \prime}$ SE. Since its discovery (Fairall 1977), Fairall 9 has been extensively studied in various spectral domains (Recondo-Gonzales et al. 1997). Recent X-ray observations (Reynolds et al. 1997; Nandra et al. 1997) show a power law spectrum in the $3-10 \mathrm{keV}$ range, a high energy tail and a fluorescent iron line.

According to the current paradigm for active galactic nuclei (AGNs), the nucleus of Seyfert galaxies would consist of an accretion disc surrounding a supermassive black hole (Rees 1984). Following this model, the main source of power is the release of gravitational potential energy as matter falls toward the central black hole. Much of this energy is emitted in the form of X-rays. A large fraction of the X-ray emission is directly observed as a power law

Send offprint requests to: P. Gondoin,

e-mail: pgondoin@estsa2.estec.esa.nl spectral component while the fluorescent iron $\mathrm{K} \alpha$ line and the high energy tail, which are present in Fairall 9 spectra, are most likely the result of X-ray reprocessing mechanisms by material within the inner region of an accretion disc. By analysing these spectral components in high ratio signal to noise data, the present study aims at new information about the geometry and the nature of the physical processes which operate in the immediate environment of the Fairall 9 central engine. The iron line, in particular, is an important tool for mapping the inner regions of the accretion disc. Using an ASCA observation performed in November 1993, Reynolds (1997) found that the Fe K line of Fairall 9 is broad and well fitted by a line emission model from a relativistic accretion disc. This may be the best evidence for the existence of a massive black hole in the nucleus of Fairall 9. It is worth noting that such evidence is not found in all AGNs. In some objects, lines are narrower than $1000 \mathrm{~km} \mathrm{~s}^{-1}$ across and vary in flux far more slowly than the continuum, suggesting that they are made far from a putative central black hole (Guainazzi et al. 1998; Weaver et al. 1996; Yaqoob et al. 2000).

In the soft energy range, the X-ray spectra of many AGNs deviate from a simple power law continuum 
absorbed by Galactic material. Below $2 \mathrm{keV}$, ROSAT observations of Fairall 9 suggest the existence of a soft excess (Pounds et al. 1994) and the possible presence of an absorption edge (Piro et al. 1997). The presence of a soft X-ray excess has been established in a large fraction of Seyfert galaxies (Turner \& Pounds 1989; Walter \& Fink 1993). A significant fraction of type 1 active galactic nuclei (Reynolds 1997) also show K-shells absorption edges of warm oxygen (O VII and O VIII) characteristic of optically thin, photoionized material along the line of sight to the central engine, the so-called warm absorber. The limitation in bandwidth and resolution of previous instruments and the presence of several components in the X-ray spectrum make the origin of the soft excess and warm absorber still unclear. A good determination of the shape of the intrinsic power law which should take into account the presence of a reflection component at high energies is particularly important for measuring the soft X-ray excess. In this respect, the present study of Fairall 9 benefits from the large $0.3-12 \mathrm{keV}$ spectral band coverage of the XMMNewton space observatory, from the high signal provided by the huge collecting area of its X-ray telescopes and from the good spectral resolution of its CCD cameras.

In this paper, we report the analysis results of an observation of Fairall 9 performed in July 2000. Section 2 details the observation and data reduction procedures. Section 3 presents the integrated flux measurements and their temporal behaviour during the observation. Section 4 describes the spectral analysis which is performed in two steps including first a characterization of the reflection components above $2 \mathrm{keV}$ and then an analysis of the soft $(0.3-2 \mathrm{keV})$ energy range. The analysis results are discussed in Sect. 5 within the frame of recent accretion disc models.

\section{Observations and data reduction}

Fairall 9 was observed by the XMM-Newton space observatory (Jansen et al. 2001) between 2000 July 5 23:03:36 (UT) and 2000 July 6 07:06:54 (UT) for a usable exposure time of $28.8 \mathrm{ksec}$. The satellite observatory uses three grazing incidence telescopes which provide an effective area higher than $4000 \mathrm{~cm}^{2}$ at $2 \mathrm{keV}$ and $1600 \mathrm{~cm}^{2}$ at $8 \mathrm{keV}$ (Gondoin et al. 2000). Three CCD EPIC cameras (Turner et al. 2001) at the prime focus of the telescopes provide imaging in a 30 arcmin field of view and broadband spectroscopy with a resolving power of between 5 and 60 in the energy band 0.1 to $15 \mathrm{keV}$. Two identical RGS reflection grating spectrometers behind two of the three X-ray telescopes allow high resolution $(E / \Delta E=100$ to 500) measurements in the soft X-ray range (0.3 to $2.1 \mathrm{keV}$ ) with a maximum effective area of about $140 \mathrm{~cm}^{2}$ at $0.83 \mathrm{keV}$ (den Herder et al. 2001).

Fairall 9 observations were conducted with the EPIC $\mathrm{p}-\mathrm{n}$ camera operating in full frame mode and with the two EPIC MOS cameras operating in small window mode. RGS spectra were recorded simultaneously. "Medium" thickness aluminum filters were used in front of the EPIC

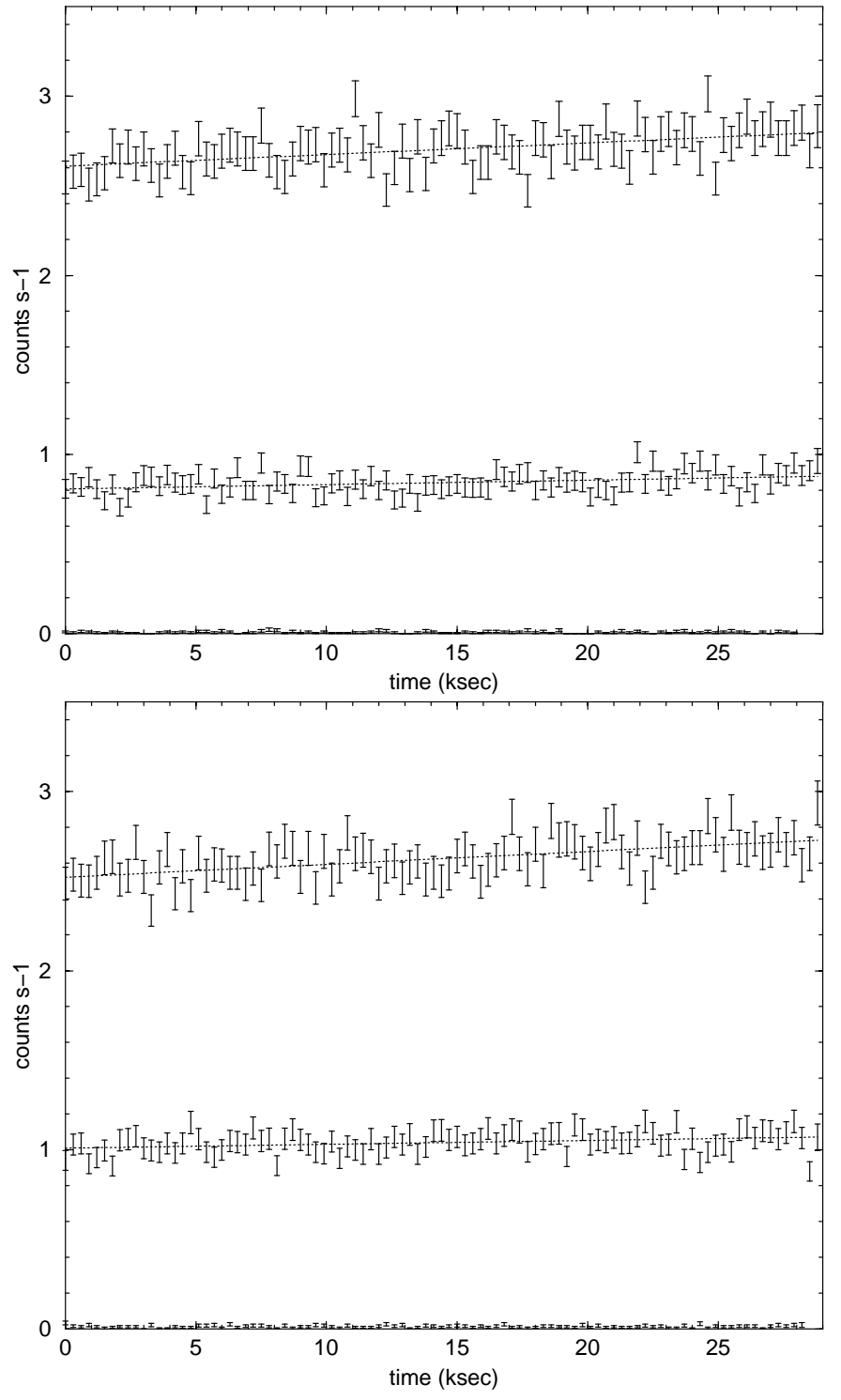

Fig. 1. Light curves of Fairall 9 simultaneously obtained with the EPIC p-n (top) and EPIC MOS (bottom) cameras in the $0.6-2 \mathrm{keV}$ (upper curve) and in the $2-10 \mathrm{keV}$ (middle curve) energy bands. The lower curves (almost invisible) show the noise contribution within the $0.6-10 \mathrm{keV}$ band. The events are binned in $300 \mathrm{~s}$ time intervals. The slight and steady rate increase within the $29 \mathrm{ksec}$ observation time is illustrated by the dotted lines obtained by linear regressions applied to the light curves.

CCD cameras to reject visible light. Processing of the raw event dataset was performed using the "emproc", "epproc" and "rgsproc" pipeline tasks of the XMM-Newton Science Analysis System (SAS version 5.0). The EPIC source spectra were built from photons detected within 60 arcsec radius from target boresight in the MOS cameras. Due to the proximity of CCD edges, a smaller 40 arcsec extraction window was used for the $\mathrm{p}-\mathrm{n}$ data. Large extraction windows could be selected because of the extremely low background rate in the detectors. They minimize the loss of source counts due to X-ray scattering of high energy photons onto the X-ray mirror surfaces. The 
background was estimated on the same CCD chips within windows of the same size which were offset by 2.5 arcmin from the source centroid position.

The Pulse-Invariant (PI) spectra were rebinned such that each resultant MOS channel had at least 50 counts per bin and each $\mathrm{p}-\mathrm{n}$ channel had at least 120 counts per bin. $\chi^{2}$ minimization was used for spectral fitting. All such fits were performed using the XSPEC package (v11). We used response matrices provided by the EPIC PI institutes. MOS 1 and MOS 2 spectra were fitted together while the $\mathrm{p}-\mathrm{n}$ spectrum was analysed separately. This approach allows us to quantify the uncertainties on derived parameters associated with the analysis procedure, the instrument calibration and the intrinsic property of the target. The analysis was conducted in the $0.3-10 \mathrm{keV}$, 0.6-10 keV and 0.3-12 keV energy bands for the MOS 1, MOS 2 and $\mathrm{p}-\mathrm{n}$ data, respectively. The analysis of MOS 2 was performed at energies higher than $0.6 \mathrm{keV}$ to avoid calibration uncertainties of the small window mode of the MOS 2 camera at the time of the analysis. Due to the greater sensitivity of the EPIC $\mathrm{p}-\mathrm{n}$ camera at high energies, the analysis of the $\mathrm{p}-\mathrm{n}$ data could be extended up to $12 \mathrm{keV}$.

\section{Integrated flux and temporal behaviour}

The spectral analysis of the Fairall 9 MOS and $\mathrm{p}-\mathrm{n}$ data (see Sect. 4) yields $0.5-2 \mathrm{keV}$ and $2-10 \mathrm{keV}$ band fluxes of $F_{\mathrm{LE}}=9.5 \pm 1.5 \times 10^{-12} \mathrm{erg} \mathrm{cm}^{-2} \mathrm{~s}^{-1}$ and $F_{\mathrm{HE}}=15.6 \pm 3.3 \times 10^{-12} \mathrm{erg} \mathrm{cm}^{-2} \mathrm{~s}^{-1}$, respectively . These correspond to luminosities of $L_{\mathrm{LE}}=3.8 \times 10^{41} \mathrm{erg} \mathrm{s}^{-1}$ and $L_{\mathrm{HE}}=6.2 \times 10^{41} \mathrm{ergs} \mathrm{s}^{-1}$ for $z=0.0461$ and $H_{0}=75 \mathrm{~km} \mathrm{~s}^{-1} \mathrm{Mpc}^{-1}$.

After correction from encircled energy fraction in the respective extraction windows, the $\mathrm{p}-\mathrm{n}$ and MOS count rates are consistent with the $\mathrm{p}-\mathrm{n}$ camera having a slightly higher sensitivity that the combined EPIC MOS 1 and 2 cameras. Count rates are low enough for pile-up effects to be negligible both in the $\mathrm{p}-\mathrm{n}$ camera and in the two MOS cameras which were operating in small window mode. A linear regression to the light curves indicates (see Fig. 1) a slight variation of the source flux over the $28.8 \mathrm{ksec}$ observation period. The count rates increased by $8.3 \pm$ $2.5 \%$ without any significant difference between the low $(0.6-2 \mathrm{keV})$ and high $(2-10 \mathrm{keV})$ energy bands.

A comparison between our measurements of Fairall 9 flux and those performed by previous missions is provided in Table 1. The Fairall 9 flux that we derived is significantly lower (see Fig. 2) than the first X-ray measurements performed by the Uhuru, Ariel, HEAO 1 and HEAO 2 experiments. However, gaps in the X-ray data prevent any firm conclusion about the trend evolution of Fairall 9 flux in the past 30 years.

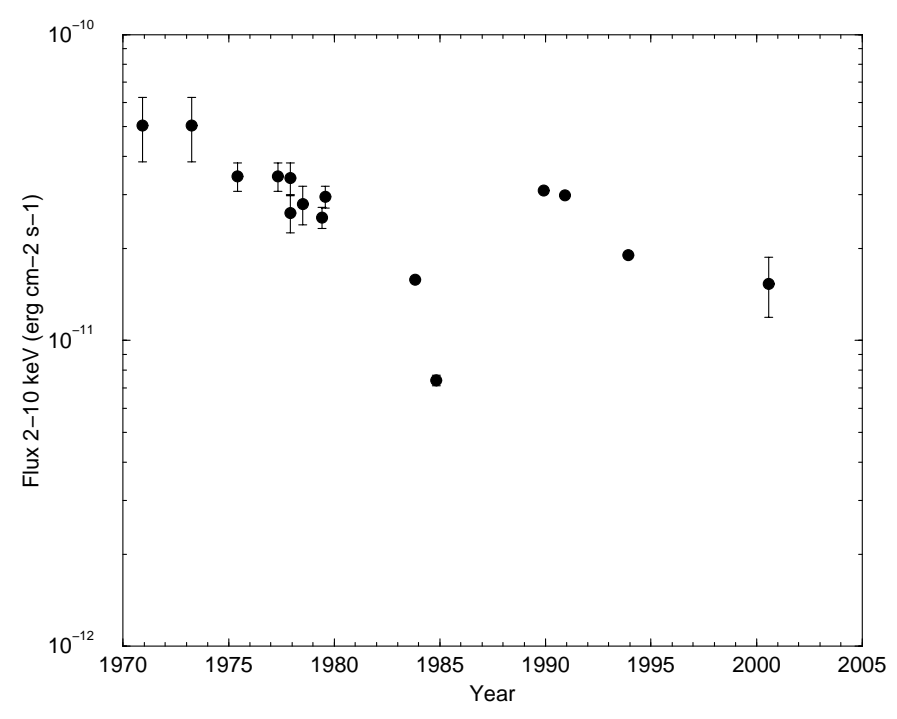

Fig. 2. The $2-10 \mathrm{keV}$ flux of Fairall 9 as a function of time in the period 1970 to 2000. References are given in Table 1. Our error estimate on Fairall 9 flux is based on the independent measurements performed by the MOS and $\mathrm{p}-\mathrm{n}$ cameras. It reflects the status of the instrument cross-calibration at the time of the analysis.

Table 1. Fairall 9 flux measurements: (1) Forman et al. 1978, (2) McHardy et al. 1981, (3) Wood et al. 1984, (4) Piccinoti et al. 1982, (5) Petre et al. 1984, (6) Morini et al. 1986, (7) Recondo-Gonzales et al. 1997, (8) Reynolds 1997, (9) Our work.

\begin{tabular}{ccc}
\hline $\begin{array}{c}\text { Experiment } \\
\text { Reference) }\end{array}$ & Date & $\begin{array}{c}2-10 \mathrm{keV} \mathrm{Flux} \\
10^{-11} \mathrm{erg} \mathrm{cm}^{-2} \mathrm{~s}^{-1}\end{array}$ \\
\hline Uhuru (1) & $1970-1973$ & $5.04 \pm 1.2$ \\
Ariel 5 (2) & $1975-1977$ & $3.44 \pm 0.37$ \\
HEAO 1 A-1 (3) & 1977 Dec. & $3.4 \pm 0.4$ \\
HEAO 1 A-2 (4) & 1977 Dec. & $2.61 \pm 0.36$ \\
HEAO 1 A-2 (4) & 1978 Jun. & $2.79 \pm 0.4$ \\
HEAO 2 (5) & 1979 May & $2.52 \pm 0.20$ \\
HEAO 2 (5) & 1979 Jul. & $2.95 \pm 0.24$ \\
EXOSAT (6) & 1983 Oct. & $1.58 \pm 0.03$ \\
EXOSAT (6) & 1984 Oct. & $0.74 \pm 0.03$ \\
GINGA (7) & 1989 Nov. & 3.09 \\
GINGA (7) & 1990 Nov. & 2.98 \\
ASCA (8) & 1993 Nov. & 1.90 \\
XMM-Newton (9) & 2000 Jul. & $1.53 \pm 0.34$ \\
\hline
\end{tabular}

\section{Spectral analysis}

\subsection{Phenomenological model of the Fe K spectral features}

We first parametrized the primary X-ray continuum of Fairall 9 in the 2 to $5 \mathrm{keV}$ energy band. The low energy restriction at $2 \mathrm{keV}$ was selected in order to minimize contamination of the Comptonization continuum by any photoelectric absorption because of a warm absorber or by any soft excess that could be present in the object. The upper $5 \mathrm{keV}$ limit was set to avoid the 5-8 keV energy band which contains the Fe $\mathrm{K}$ features and to reject any 
contribution above $8 \mathrm{keV}$ that could be associated with a high energy reflection bump.

Our initial fitting model was a single redshifted power law absorbed by a column density of $3.2 \times$ $10^{20} \mathrm{~cm}^{-2}$ to take into account the Galactic contribution to the photoelectric absorption (Dickey \& Lockman 1990). Simultaneous fitting of the MOS and $\mathrm{p}-\mathrm{n}$ cameras did not provide an acceptable $\chi^{2}$. Hence, the MOS and $\mathrm{p}-\mathrm{n}$ data were fitted separately. The results indicate that the $90 \%$ confidence ranges of the photon indices derived independently from the MOS and $\mathrm{p}-\mathrm{n}$ spectra intersect at $\Gamma=1.73$ (see Table 2).

Table 2. Power law model of Fairall 9 spectrum in the $2-5 \mathrm{keV}$ spectral range. The table provides the average of the parameters which best fit EPIC MOS and EPIC $\mathrm{p}-\mathrm{n}$ spectra with reduced $\chi^{2}$ of 1.01 and 0.90 respectively.

\begin{tabular}{cc}
\hline Model parameters & \\
\hline \hline$N_{\mathrm{H}}\left(\mathrm{cm}^{-2}\right)$ & $3.2 \times 10^{20}$ \\
Photon index & $1.73 \pm 0.07$ \\
Flux $\left(\mathrm{erg} \mathrm{s}^{-1} \mathrm{~cm}^{-2}\right)$ & $7.4 \pm 1.5 \times 10^{-12}$ \\
\hline
\end{tabular}

The Fairall 9 spectrum is known to exhibit a high energy tail (Reynolds 1997). Various authors consider that $\mathrm{X}$-ray reflection upon an optically thick slab of cool material (George \& Fabian 1991) could explain such a tail. In the case of Fairall 9, the material in the slab could be partially ionized in view of the plausible proximity of the intense X-ray source emitting the primary continuum. A reflected spectrum is expected to leave a bump between 20 and $50 \mathrm{keV}$ due to the combination of photoelectric opacity at low energy and Compton recoil at the high energy end of the spectrum. This high energy band was not accessed during our observation. However, the EPIC cameras are sensitive up to about $12 \mathrm{keV}$ and a major part of the absorptive opacity from $7-20 \mathrm{keV}$ is Fe-K edge opacity. A significant fraction of incident photons within this energy range are expected to give rise to the iron K-shell fluorescent line due to the high abundance and fluorescence yield of iron.

An emission feature is indeed observed around $6.4 \mathrm{keV}$ within the EPIC MOS and EPIC p-n spectra. Also, comparison of the data to the $2-5 \mathrm{keV}$ power law model extrapolated to the high energy range shows a broad excess excess emission above the Fe $\mathrm{K}$ line up to an energy of about $7.2 \mathrm{keV}$ (see Fig. 3) which suggests the presence of an absorption edge. Based on these features, we fit the EPIC data in the high energy range above $5 \mathrm{keV}$ by a phenomenological model consisting of a power law continuum, a Gaussian line and an absorption edge. The best fit models to the EPIC MOS and $\mathrm{p}-\mathrm{n}$ data consistently point to the existence of an iron $\mathrm{K} \alpha$ fluorescence line at $6.4 \mathrm{keV}$ and an absorption edge at $7.6 \mathrm{keV}$ in the source rest frame (see Table 3).

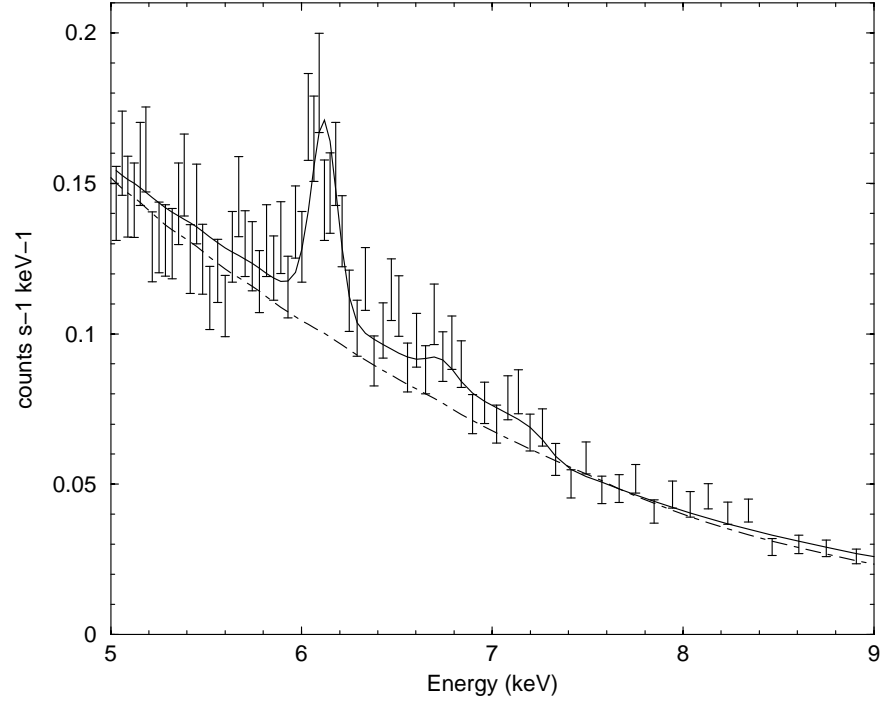

Fig. 3. Comparison of $\mathrm{p}-\mathrm{n}$ data with a phenomenological model (bold solid line) of the Fe K complex consisting of two redshifted Gaussians and an absorption edge. The ratio of the Gaussian normalisation factors has been fixed to the theoretical value of the $\mathrm{Fe} \mathrm{K} \beta$ to $\mathrm{Fe} \mathrm{K} \alpha$ line ratio. The best fit power law model to the data in the $2-5 \mathrm{keV}$ spectral region is represented by a dot-dashed line.

Table 3. Gaussian line and absorption edge parameters of a phenomenological model of Fairall 9 spectrum in the $5-10 \mathrm{keV}$ spectral band. For energies greater than the edge energy $E_{0}$, the absorption edge is defined as $\exp \left[-\tau\left(E(1+Z) / E_{0}\right)^{-3}\right]$ where $\tau$ is the absorption depth at $E_{0}$. The table provides the average of the parameters which best fit EPIC MOS and EPIC $\mathrm{p}-\mathrm{n}$ spectra with reduced $\chi^{2}$ of 1.02 and 1.0 respectively. Parameter ranges are given at a $90 \%$ confidence level.

\begin{tabular}{cc}
\hline Model parameters & \\
\hline \hline Edge energy $(\mathrm{keV})$ & $7.64_{-0.36}^{+0.21}$ \\
Edge abs. depth & $0.18_{-0.10}^{+0.18}$ \\
\hline Line energy $(\mathrm{keV})$ & $6.38 \pm 0.03$ \\
Line $\sigma(\mathrm{keV})$ & $0.05 \pm 0.05$ \\
Equivalent width $(\mathrm{keV})$ & 0.12 \\
\hline
\end{tabular}

The iron $\mathrm{K} \alpha$ fluorescence line consists of two components $\mathrm{K} \alpha_{1}$ and $\mathrm{K} \alpha_{2}$ at $6.404 \mathrm{keV}$ and $6.391 \mathrm{keV}$ respectively for Fe I and a branching ratio of 2:1 (Bambynek et al. 1972). The natural width of the lines $(\Delta E \approx 3.5 \mathrm{eV})$ and any broadening due to thermal motions of the emitting atoms $\left(\Delta E(\mathrm{eV}) \approx 0.4\left(T / 10^{6}\right)^{1 / 2}\right)$ are negligible compared to the energy resolution (155 eV FWHM) of the EPIC cameras. Also, no distinction between $\mathrm{K} \alpha_{1}$ and $\mathrm{K} \alpha_{2}$ photons can be made. The iron $\mathrm{K} \alpha$ line energy is an increasing function of ionization state. It rises slowly from $6.40 \mathrm{keV}$ in Fe I to $6.45 \mathrm{keV}$ in Fe XVII (neonlike) and then increases steeply with the escalating number of vacancies in the L-shell to $6.7 \mathrm{keV}$ in Fe XXV and $6.9 \mathrm{keV}$ in Fe XXVI (House 1969; Makishima 1986). Hence, the measured energy position of the Fairall 9 Fe K 
line at $6.38 \pm 0.03$ indicates that iron is in a low state of ionization.

The position in energy of the Fe absorption edge is also function of the ionization stage of the Fe element. Aside from any blurring effect by the detector spectral response, the iron $\mathrm{K}$ edge is not expected to be sharp due to electron scattering and by the presence of the iron $K \beta$ line. Hence, the exact measurement of its position is difficult. In the case of $\mathrm{Fe} \mathrm{I}$, the K-shell absorption edge is at an energy $E_{\mathrm{K}}=7.1 \mathrm{keV}$, rising to $7.8 \mathrm{keV}$ for Fe XVIII, and $9.3 \mathrm{keV}$ for Fe XXVI (Morita \& Fujita 1983). The measured energy position of the Fairall 9 Fe K absorption edge at $7.64_{-0.36}^{+0.21}$ is consistent with a reflection model from cold optically thick material. However, in the case of Fairall 9, Cold means that iron could be in different states of ionization ranging from neutral to Fe XVII. For other abundant elements, this would mean that oxygen is not fully ionized although $\mathrm{H}$ and $\mathrm{He}$ may be ionized.

\subsection{Reflection model of the continuum above $2 \mathrm{keV}$}

Since, the phenomenological model indicates a position of an iron $\mathrm{K}$ edge above $7.1 \mathrm{keV}$, we initially replace the phenomenological model by a PEXRIV model (Magdziar \& Zdziarski 1995) combined with a redshifted Gaussian component to represent the emission line. Fitting test performed for solar abundance provided a reduced $\chi^{2}$ very closed to unity for both MOS and $\mathrm{p}-\mathrm{n}$ data. In both case however, the best fit disc temperature indicated a low value temperature pegged to the $10000 \mathrm{~K}$ lower limit of the model.

Hence, we replaced the PEXRIV model of a power law spectrum reflected from ionized material by the PEXRAV model of a power law spectrum reflected from neutral material (Magdziarz \& Zdziarsk 1995). The abundance, the energy cut-off and the disc inclination were frozen while the power law index and the amount of reflection were left free. Table 4 (Model A) lists the fitting parameters obtained when the abundance of elements heavier than $\mathrm{He}$ are set to the solar abundance values defined by Anders \& Grevesse (1989). Under the solar abundance hypothesis, a reflection fraction greater than 1 is obtained suggesting that the reflected spectrum could be enhanced. A number of scenarios have been proposed that could explain such a phenomenon including geometrical effects where the direct emission is partially obscured, motions of the source (Reynolds \& Fabian 1997) or light-bending effects (Martocchia \& Matt 1996). However, the reflection fraction is poorly constrained and the amount of reflection is strongly coupled to the elemental abundances (see e.g. George \& Fabian 1991; Matt et al. 1991). Table 4 (Model $\mathrm{B}$ ) gives the best fit parameters obtained when the scaling factor for reflection is set to 1, i.e. when the primary X-ray source is assumed to lay above the accretion disc subtending a solid angle of $2 \pi$ sr. In that case, an acceptable fit to the data is obtained for subsolar abundances. In spite of the degeneracy between reflected fraction and material abundances, both models lead to consistent measurements of the photon spectral index of the primary continuum. The measured index of 1.80 is slightly higher than the values derived in the $2-5 \mathrm{keV}$ energy band (see Table 2 ). The higher value arises as a result of the reflected spectrum masking a steeper intrinsic power law slope even at energies lower than $5 \mathrm{keV}$. This effect has to be taken into account in the characterization of the soft-excess contribution when extrapolating the primary power law continuum to energies lower than $2 \mathrm{keV}$.

Table 4. PEXRAV + ZGAUSSIAN models of Fairall 9 spectrum in the $2-10 \mathrm{keV}$ spectral band. The left column (Model A) describes the best fit model obtained when the abundances are frozen to the solar values. The right column (Model B) gives the best fit parameters obtained when the reflected fraction is set to unity. The parameters of the Gaussian model are identical to those provided in Table 3 . The table provides the average of the parameters which best fit EPIC MOS and EPIC $\mathrm{p}-\mathrm{n}$ spectra with reduced $\chi^{2}$ of 1.01 and 0.96 respectively. Parameter ranges are given at a $90 \%$ confidence level.

\begin{tabular}{ccc}
\hline Model parameters & Model A & Model B \\
\hline \hline Photon index & $1.79_{-0.12}^{+0.15}$ & $1.81_{-0.13}^{+0.24}$ \\
Cutoff energy & $100 \mathrm{keV}$ & $100 \mathrm{keV}$ \\
Reflected fraction & $2.0_{-1.0}^{+2.0}$ & 1.0 \\
Redshift & 0.0461 & 0.0461 \\
Abundance & 1.0 & $0.5 \pm 0.5$ \\
Inclination & $26^{\circ}$ & $26^{\circ}$ \\
\hline
\end{tabular}

The profiles of lines emitted from an accretion disc can be strongly modified by relativistic effects (Fabian et al. 1989; Stella 1990). Replacing the Gaussian model of the Fe K line by a DISKLINE (Fabian et al. 1989) or a LAOR (Laor 1991) model led to a significant degradation of the fitting statistics. The Fe $\mathrm{K}$ line profile of Fairall 9 is not much broader (see Table 3) than the EPIC spectral response at $6.4 \mathrm{keV}$. According to the computation of Matt et al. (1992a,b), the Fe K $\alpha$ line produced by an accretion disc is expected to be narrowest when the disc is seen face on. However, even at small inclination angles, the contribution of gravitational and second order Doppler shifts to the line width can be substantial, obviously depending of the inner radius of the disc. Since the Fe K line observed in the Fairall 9 spectrum is narrow, it is unlikely that it includes any significant contribution from material with relativistic motion in a strong gravitational field.

The equivalent width $W_{\alpha}$ of the $\mathrm{K}_{\alpha}$ line depends on several parameters (Matt et al. 1991), namely the iron abundance, the inclination and size of the disc, the geometry of the primary photon source. Hence, a determination of iron abundance from $W_{\alpha}$ is subject to the uncertainties on the other parameters not to mention the complications associated with the effects of time delays in the response of the reprocessed radiation to variations in the primary source. In the case of a semi-isotropically illuminated slab 
of cold matter with cosmic elemental abundance, George \& Fabian (1991) calculated that $W_{\alpha}$ varies approximately linearly from 150 to $90 \mathrm{eV}$ for power slope ranging from 1.2 to 2.1 , respectively. The measured $120 \mathrm{eV}$ equivalent width of the $\mathrm{Fe} \mathrm{K}$ line and the slope index of the Fairall 9 Comptonization continuum seems consistent with such a model. However, the above $W_{\alpha}$ values were calculated by integrating the escaping fluorescent photons over $2 \pi$ steradian. They are therefore not necessarily what an observer detects under a given disc aspect angle. In the case of a centrally illuminated disc seen face on (George \& Fabian 1991), the height of the source with respect to the inner disc radius contributes very significantly to reduce the equivalent width of the $\mathrm{Fe} \mathrm{K}_{\alpha}$ line, thus preventing any conclusion regarding iron abundance.

\subsection{The soft (0.3-2 keV) energy range}

A reflection model by a cool slab of material in a low ionization state provides an adequate description of the Fairall 9 spectrum above $2 \mathrm{keV}$. In order to characterize the overall shape of EPIC spectra, we extended the analysis to the soft energy range. A lower limit to the analysis was set to $0.3 \mathrm{keV}$ for the MOS 1 and $\mathrm{p}-\mathrm{n}$ cameras and to $0.6 \mathrm{keV}$ for the MOS 2 camera due to calibration uncertainties of the MOS 2 response at low energy in window mode. Comparisons of the MOS and $\mathrm{p}-\mathrm{n}$ spectra with the PEXRAV + ZGAUSS model (see Table 4) established above $2 \mathrm{keV}$ give large residuals at soft energies.

This led us to consider additional redshifted components including a black body model, a Bremsstrahlung model (Kellog et al. 1975) and a power law model. These components were combined with neutral galactic absorption using the cross-sections of Morrison \& McCammon (1983). Two values of the galactic hydrogen column density were initially considered, namely $N_{\mathrm{H}}=2.8 \times 10^{20} \mathrm{~cm}^{-2}$ (Piro et al. 1997) and $N_{\mathrm{H}}=$ $3.2 \times 10^{20} \mathrm{~cm}^{-2}$ (Nandra et al. 1997) with solar abundances. Since the fitting parameters did not differ significantly when using these different $N_{\mathrm{H}}$ values, spectral fitting was then conducted assuming galactic absorption with solar abundance and $N_{\mathrm{H}}=3.0 \times 10^{20} \mathrm{~cm}^{-2}$. The power law model gives unacceptable fit to both MOS and $\mathrm{p}-\mathrm{n}$ data. The redshifted thermal Bremsstrahlung model provides a good description of both $\mathrm{p}-\mathrm{n}$ and MOS data. However, Bremsstrahlung temperatures were found in the range 0.6 to $0.9 \mathrm{keV}$. At these temperatures and for solar abundances, the spectrum would be dominated by line emission blends and recombination edges. Thus, the Bremsstrahlung model for this temperature regime was regarded as physically inconsistent.

The ratio of the $\mathrm{p}-\mathrm{n}$ data to the best fit of the data by the PEXRAV + blackbody model still presents a residual emission feature which deviates significantly from the continuum at $0.56 \mathrm{keV}$ (see Fig. 5). In order to parametrize this residual emission, we added a redshifted Gaussian
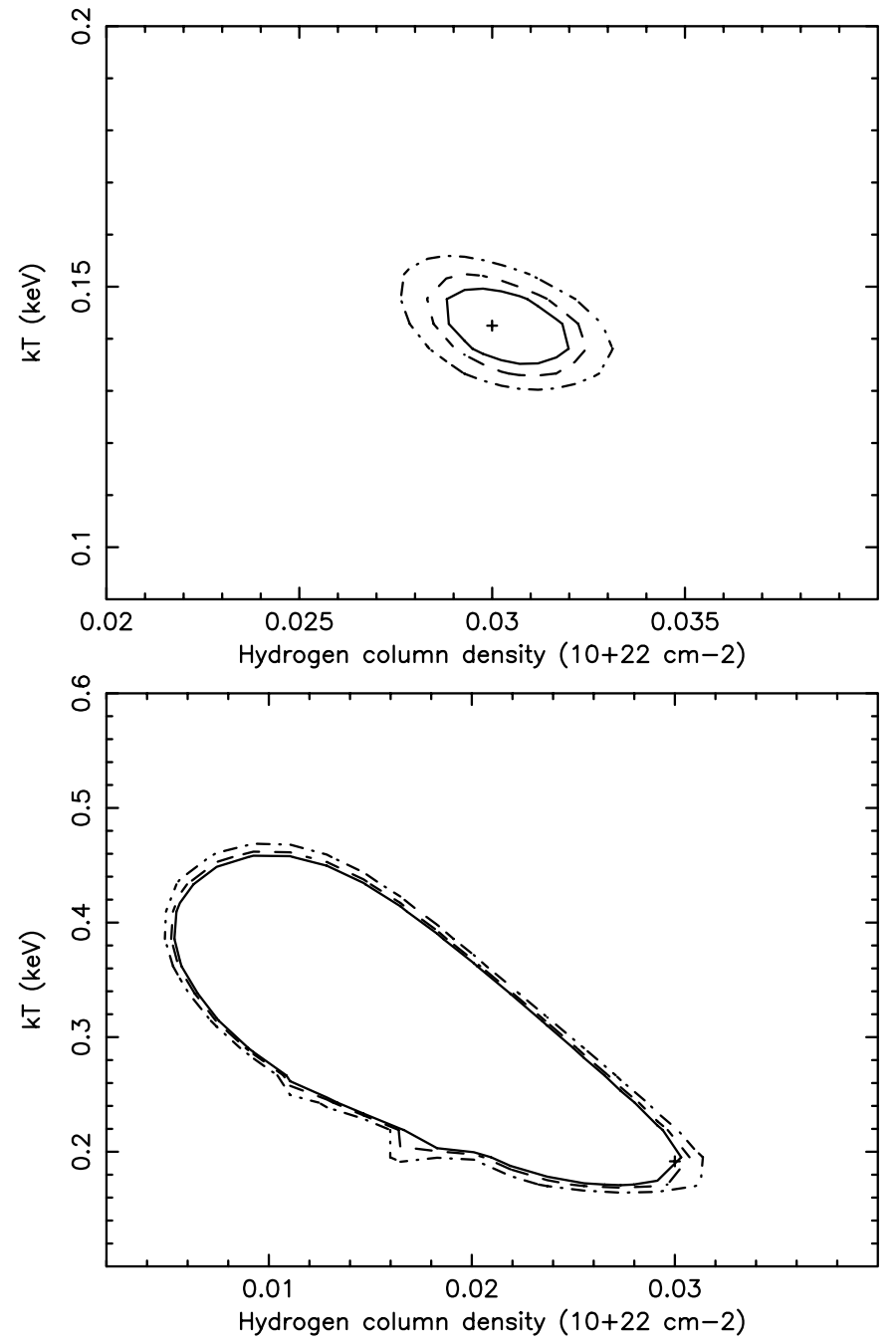

Fig. 4. EPIC $\mathrm{p}-\mathrm{n}$ (top) and MOS (bottom) confidence contours (corresponding to 68, 90 and 95 percent confidence levels) for blackbody temperature versus galactic hydrogen column density.

to the PEXRAV + blackbody model. By adding a redshifted Gaussian, the $\chi^{2}$ decreases by $\delta \chi^{2}=-21$ for 613 degrees of freedom. A best fit line position is found at $0.60 \pm 0.02 \mathrm{keV}$ in the source rest frame. It is worth comparing this energy with the line positions of ionized elements. Among elements with a significant abundance, oxygen is the best candidate. OVII lines are indeed present around $0.57 \mathrm{keV}$ and an OVIII $\mathrm{K} \alpha$ recombination line is located at $0.65 \mathrm{keV}$. Hence, the position of the residual emission detected in the EPIC $\mathrm{p}-\mathrm{n}$ spectrum of Fairall 9 could be related to the presence of oxygen in a high state of ionization along the line of sight. In order to check this hypothesis, we applied the same procedure to the MOS data. In that case, the addition of an emission line to the best PEXRAV reflection + blackbody model did not improve the fitting quality. The higher spectral resolution of the RGS spectrometers on board XMM-Newton is well suited to disentangle emission line features from absorption edges and to detect the presence of a warm absorber in the 0.3 to $2.1 \mathrm{keV}$ energy band. The RGS average spectrum (see Fig. 6) of Fairall 9 shows an absorption edge 
Table 5. Best fit models of Fairall 9 spectrum in the 0.3-10 keV (MOS 1), 0.6-10 keV (MOS 2) and 0.3-12 keV $(\mathrm{p}-\mathrm{n})$ spectral range. The table provides the average of the parameters which best fit EPIC MOS and EPIC $\mathrm{p}-\mathrm{n}$ spectra with reduced $\chi^{2}$ of 1.21 and 1.01 respectively. The parameters of the Gaussian line at low energies were derived from the $\mathrm{p}-\mathrm{n}$ spectrum.

\begin{tabular}{cc}
\hline Model parameters & \\
\hline \hline Absorption model & \\
$N_{\mathrm{H}}\left(\mathrm{cm}^{-2}\right)$ & $3.0 \times 10^{20}$ \\
$\times$ & \\
Reflection model & see Table 4 \\
+ & \\
Fe K line model & \\
$E(\mathrm{keV})$ & $6.38 \pm 0.03$ \\
+ & \\
blackbody model & $0.17_{-0.07}^{+0.09}$ \\
$T(\mathrm{keV})$ & \\
+ & \\
Gaussian line model & $0.60 \pm 0.02$ \\
$E(\mathrm{keV})$ & 0.050 \\
$\sigma(\mathrm{keV})$ & 11.4 \\
Eq. Width $(\mathrm{eV})$ & \\
\hline
\end{tabular}

at $0.54 \mathrm{keV}$ consistent with galactic absorption by neutral oxygen. No evidence is found for K-shells absorption edges at $0.74 \mathrm{keV}$ (OVII) and $0.85 \mathrm{keV}$ (OVIII) characteristic of the presence of ionized material along the line of sight. The RGS effective area is not large enough to constrain the spectral model of Fairall 9. The achieved signal to noise ratio does not permit to confirm the existence of the emission feature detected in the EPIC $\mathrm{p}-\mathrm{n}$ spectrum at $0.56 \mathrm{keV}$. Hence, we cannot conclude that this feature is unambiguously related to an oxygen emission line of cosmic origin.

The existence of high temperature $\left(T \geq 10^{6} \mathrm{~K}\right)$ plasmas in Fairall 9 is suggested by the best fit blackbody models to the EPIC spectra. EPIC $\mathrm{p}-\mathrm{n}$ and MOS confidence contours (see Fig. 4) for blackbody temperature versus hydrogen column density overlap at $95 \%$ confidence level in the domain $2.8 \times 10^{20} \mathrm{~cm}^{-2}<N_{\mathrm{H}}<$ $3.2 \times 10^{20} \mathrm{~cm}^{-2}$ and $0.15 \mathrm{keV}<k T<0.16 \mathrm{keV}$. Figure 4 shows that the temperatures are well constrained and supports the hypothesis that the neutral absorption at low energies is mostly of galactic origin. The blackbody spectral component is obtained by comparison with best fit reflection models constrained in the $2-10 \mathrm{keV}$ energy bands. The slight difference between the best fit slope indices measured on MOS and $\mathrm{p}-\mathrm{n}$ data in that mid-energy range is amplified in the soft energy range. This leads to different flux estimate of Fairall 9 primary continuum below $1 \mathrm{keV}$. This uncertainty impacts on the parametrization accuracy of the soft excess contribution but illustrates the advantage of our parallel analysis of the MOS and $\mathrm{p}-\mathrm{n}$ data. This approach enables us to quantify the uncertainties on derived parameters, such as the soft excess flux, which result from the analysis procedure, the instrument calibration and the intrinsic property of the target.

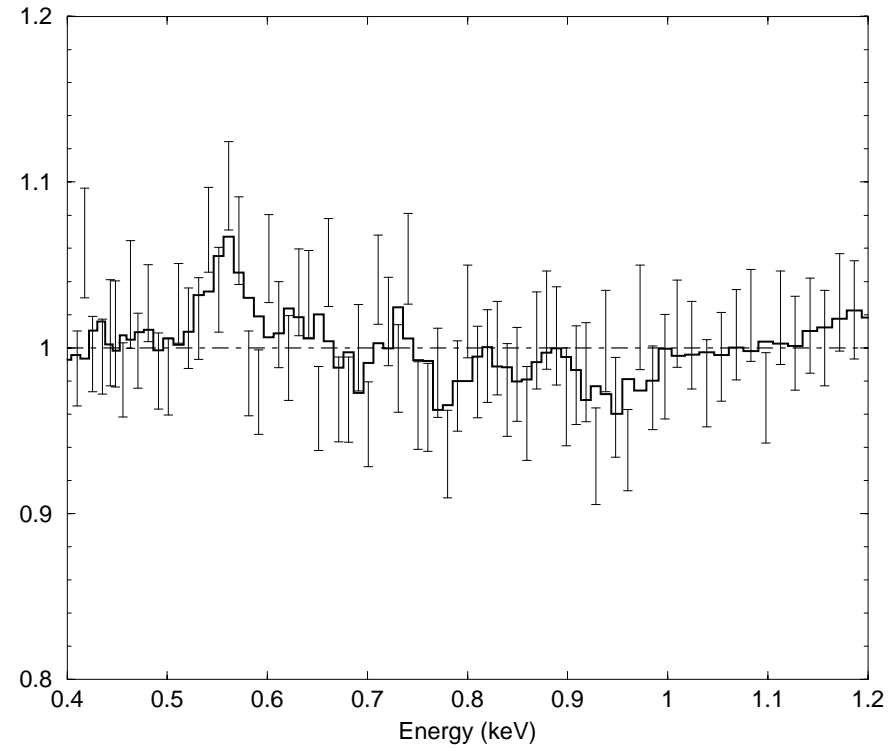

Fig. 5. Ratio of the $\mathrm{p}-\mathrm{n}$ data to the best fit reflection + blackbody model with galactic absorption by neutral gas. The solid line represents a running average over 4 bins with a minimum of 1000 counts per bin. A significant residual emission peaks at $0.56 \mathrm{keV}$ in the observer rest frame.

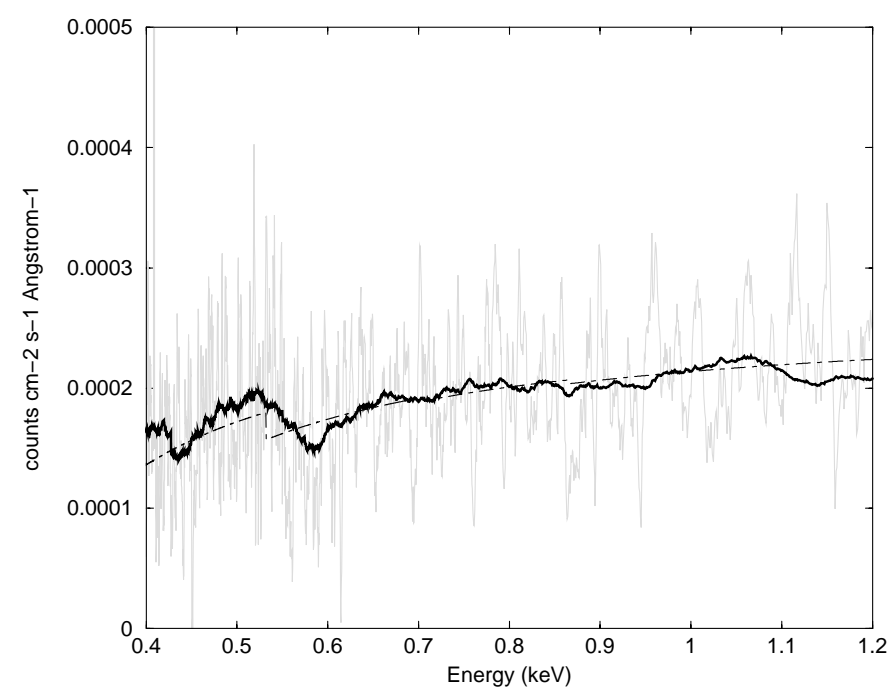

Fig. 6. First order averaged spectrum of RGS compared with a phenomenological model consisting of a redshifted power law absorbed by galactic neutral gas. The solid line is a running average performed to vizualize the OI absorption edge at $0.54 \mathrm{keV}$.

The independent measurements performed with the MOS and $\mathrm{p}-\mathrm{n}$ cameras provide Fairall 9 fluxes corrected from galactic absorption of $9.5 \pm 1.5 \times 10^{-12} \mathrm{erg} \mathrm{cm}^{-2} \mathrm{~s}^{-1}$ and $15.6 \pm 3.3 \times 10^{-12} \mathrm{erg} \mathrm{cm}^{-2} \mathrm{~s}^{-1}$ in the $0.5-2 \mathrm{keV}$ and 2-10 keV energy band, respectively.

\section{Discussion}

\subsection{The reflection components}

The high signal to noise ratio of the MOS and $\mathrm{p}-\mathrm{n}$ spectra shows an emission line at $6.4 \mathrm{keV}$ and an absorption edge 
around $7.6 \mathrm{keV}$ that has not been reported before. The excellent fit obtained with reflection models above $2 \mathrm{keV}$ indicates that these imprinted features are likely the result of partial reprocessing of a primary X-ray continuum by cold, optically thick material.

The underlying physical processes responsible for the primary non-thermal emission in radio-quiet AGNs are currently unclear. One feasible explanation is that the emission is the result of inverse Compton scattering of low-energy photons by relativistic electrons. The Comptonization process could take place either on thermal electrons in an optically thin corona, on thermal electrons in the innermost part of an accretion disc or on nonthermal electrons at the base of a jet like flow. Although the geometry of the region emitting the primary X-ray spectrum is not known, the general consensus is that the power law component originates from an extended corona above an accretion disc (Haardt \& Matt 1993).

Within the frame of such a model, the Fe K $\alpha$ emission and the absorption edge in Fairall 9 spectrum would be produced by Compton reflection of the primary continuum onto an accretion disk. Possible contributions to these reflection components may come from material within the accretion disk itself (Pounds et al. 1990) or from matter further out (Krolik et al. 1994; Ghisellini et al. 1994). Many Fe K lines are observed to be several tens of thousands of $\mathrm{km} \mathrm{s}^{-1}$ broad and significantly redshifted (Mushotzky et al. 1995; Tanaka et al. 1995; Page et al. 2001). Orbital motion deep in a relativistic gravitational potential seems to be the only interpretation for line profiles of that sort. Other AGNs show narrow Fe $\mathrm{K}$ lines (Reeves et al. 2001a; Yaqoob et al. 2001). Also, a unique iron $\mathrm{K}$ emission line profile with two distinct components has been recently reported (Reeves et al. 2001b). The Fairall $9 \mathrm{Fe} \mathrm{K}$ emission line that we detected is narrow suggesting contribution from material in low ionization states relatively far from a putative central black hole.

It is however worth noting that there are different ways to explain the lack of a relativistic Fe K line. One possibility is that the inner accretion disk does not exist as such but is replaced by an X-ray hot flow. Stable solutions of such an accretion flow model have been found by Narayan \& Yi (1995) which include advective as well as radiative cooling. An alternative solution is that the inner disc is present but cannot be seen in the reflected spectrum e.g. because it is fully ionized. Our result contrasts with the broad Fe K fluorescent line detected by Reynolds (1997) in a Fairall 9 spectrum obtained in November 1993. At this time, Fairall 9 flux was slightly higher (see Fig. 2) and its spectrum in the $2-10 \mathrm{keV}$ range was steeper $(\Gamma=1.92)$. This suggests that the equivalent width and possibly the profile of the Fe K line are variable and affected by the luminosity of the primary continuum.

The energy position of the Fe $\mathrm{K}$ line at $6.38 \pm 0.03 \mathrm{keV}$ indicates that iron is in a low state of ionization. An alternative explanation to the Fe $\mathrm{K}$ complex invokes partial covering of the X-ray source by cold, dense material. To produce a line width of about $100 \mathrm{eV}$, as observed in the present object, this medium should have a column density of iron corresponding to about $10^{23} \mathrm{H}$ atoms $\mathrm{cm}^{-2}$ (for solar abundances) in the case of homogeneous and spherical distribution (Inoue 1985). Although absorption by dense clouds cannot be excluded, reflection from an accretion disk is an appealing scenario since (i) it provides a good fit to the spectra with a small number of free parameters, (ii) it can account for several features observed in the spectrum of Fairall 9 including the UV bump (RecondoGonzales et al. 1997), the Compton reflection continuum and the soft X-ray excess that we report in this study.

The gas of the reflection slab could apparently remain essentially cool despite being exposed to an intense flux of X-rays. Such a result can be interpreted as an indication that the gas in the accretion disk is dense (Guilbert \& Rees 1988; Ferland \& Rees 1988). The detected absorption edge at $7.64 \mathrm{keV}$ has an energy position also indicating photoelectric absorption by iron atoms in low state of ionization. However, the position of the edge above $7.1 \mathrm{keV}$ indicates that atoms in different states of ionization ranging from neutral to Fe XVII contribute to the absorption. The strength of the Fe $\mathrm{K}$ absorption edge cannot be used to measure the iron abundance without assumption on the reflected fraction of the primary continuum flux. Assuming a reflection fraction of 1, the analysis of MOS and $\mathrm{p}-\mathrm{n}$ spectra indicate that the iron abundance within the cool material reflecting the primary continuum would be lower than the cosmic value. This would contrast with the overabundance suggested by the high ratio FeII(optical+UV)/MgII $\lambda 2798$ derived by RecondoGonzales et al. (1997). Further information cannot be derived from the reflection spectrum about the abundance and kinematics of the gas responsible for the reprocessing. The disc itself may be in the form of dense clouds and the source of the hard flux may be very close to the disc and even in the intercloud medium. Composite reflected spectra have been calculated for specific geometries (George \& Fabian 1991) including a semi-isotropically illuminated slab and a centrally illuminated disc. The overall shape of the reflected spectra is predicted to be similar in both cases and cannot be used to constrain the geometry of the system.

\subsection{The soft excess contribution}

A simultaneous Ginga-ROSAT observation of Fairall 9 obtained during the All Sky Survey suggested the presence of a soft excess in the spectrum of Fairall 9 (Pounds et al. 1994). The presence of a true soft X-ray emission is also indicated by the analysis of the ROSAT data by Piro et al. (1997). These authors further concluded that another feature, probably an edge, might also be present. The high signal to noise ratio of our data and the determination of the intrinsic power law spectrum of Fairall 9 in the $2-12 \mathrm{keV}$ energy band taking into account the reflection component allow us to characterize the soft X-ray excess. Confidence in the results is reinforced by the consistent 


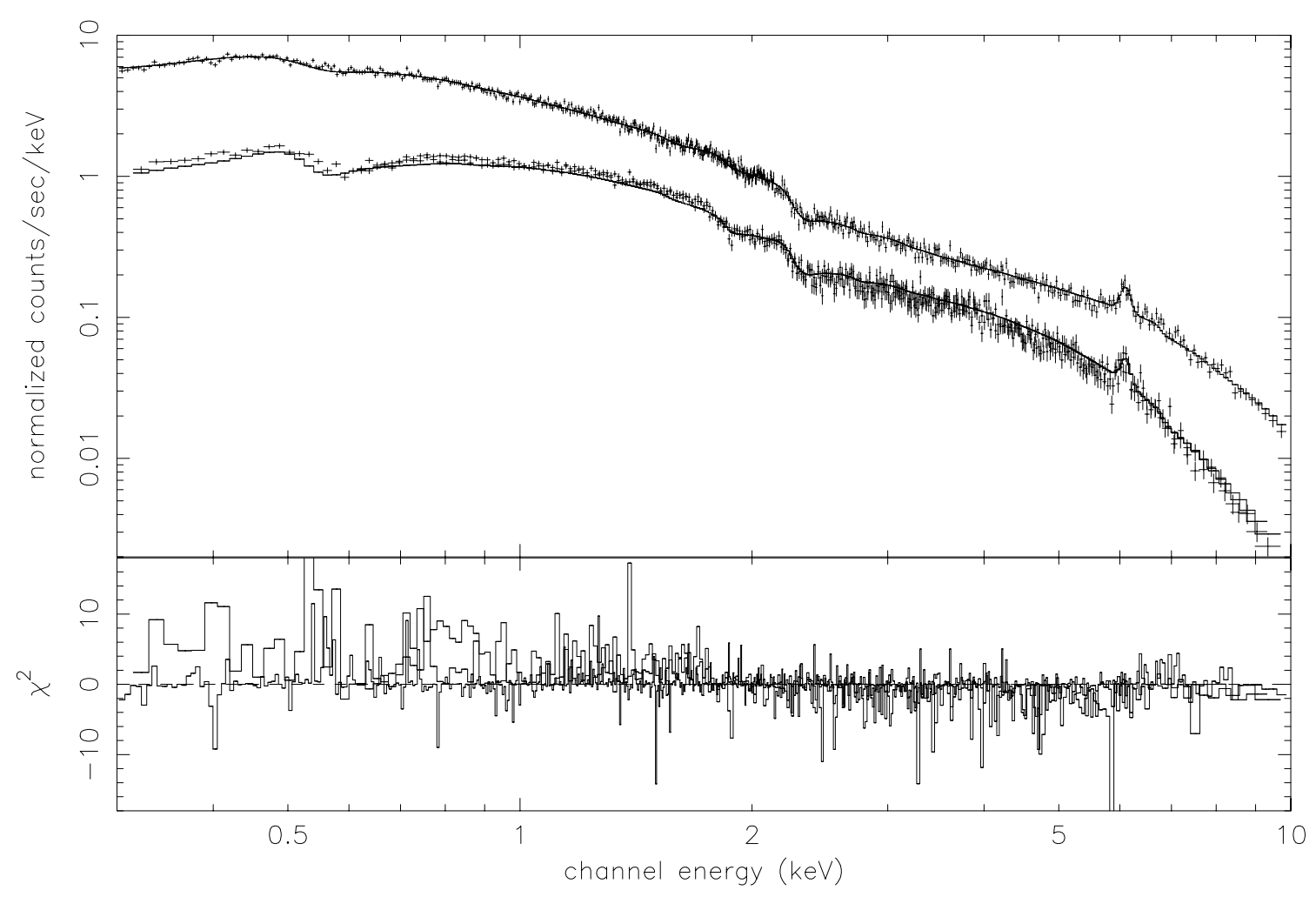

Fig. 7. EPIC $\mathrm{p}-\mathrm{n}$ (top) and MOS (bottom) spectra compared with the best fit model to the $\mathrm{p}-\mathrm{n}$ spectrum. The data and spectral fit are shown in the upper panel. The $\chi^{2}$ contributions in the lower panel indicate that the slope of MOS spectra is steeper than that of the best fit model.

models obtained from the separate analysis of the MOS and $\mathrm{p}-\mathrm{n}$ data. Our analysis concludes that, at the time of the observation, the soft component can be described by a blackbody model with a temperature $T=0.17_{-0.07}^{+0.09} \mathrm{keV}$ absorbed by neutral material with an hydrogen column density equal to the galactic value. In the low energy domain below $2 \mathrm{keV}$, we did not find evidence for the presence of absorption edges other than the one produced by galactic neutral oxygen. The flux of the soft component is $1.0 \pm 0.3 \times 10^{-12} \mathrm{erg} \mathrm{cm}^{-2} \mathrm{~s}^{-1}$ in the $0.3-1 \mathrm{keV}$ range, i.e. $14 \pm 6 \%$ of the overall Fairall 9 flux in that spectral band.

Explanations for a spectral steepening in the soft Xray band based on reflection by ionized media have been successfully applied to a few AGNs (Piro et al. 1997; Zycki et al. 1994; Ross \& Fabian 1993). According to these scenarii, the inner region of the disk could be photo-ionized by X-rays emitted from the primary continuum source. If the ionization level is such that light elements (such as He, $\mathrm{C}$ or $\mathrm{O}$ which are the main contributors to photoelectric absorption at low energies) are fully or nearly stripped of electrons, the medium becomes reflective by Thomson scattering to X-rays. Detailed models of this reflected component have been computed by Ross \& Fabian (1993) and Matt et al. (1994) for the highly ionized case and by Zycki et al. (1994) for the midly ionized case. These models typically include line emissions from OVIII $(E=0.65 \mathrm{keV})$ and OVII $(E=0.57 \mathrm{keV})$, with an equivalent width depending on the ionization parameter. If conclusive, our possible detection of an emission feature around $0.6 \mathrm{keV}$ in the EPIC $\mathrm{p}-\mathrm{n}$ spectra of Fairall 9 would reinforce the hypothesis of such a low energy reflection component.

Illumination of a cold disk by hard X-rays lead to the formation of a very hot, optically thin layer of material above the disk (Krolik et al. 1981). The two layers are in pressure equilibrium and the discontinuity in temperature and density reflects the discontinuity in cooling mechanisms. In the hot layer, Comptonization and bremstrahlung play the main roles, but closer to the cool, dense disc, atomic absorption and emission become the major cooling processes. Simulation of the spectrum resulting from such a model have been performed for various range of ionization parameters (Ross et al. 1999) assuming uniform density slabs of gas illuminated by a power law spectrum. For soft X-rays $(E<1.5 \mathrm{keV})$, results indicate that reflection effects of the primary continuum can be largely enhanced owing to Bremsstrahlung emission by the hot surface layer and "inverse Compton" upscattering of even softer photons. Depending on the primary spectrum hardness, ionization parameter and disk density, the temperature of the illuminated surface is found to be extremely high $\left(T>10^{7} \mathrm{~K}\right)$. Such high temperatures are suggested by the Bremstrahlung models of the Fairall 9 soft excess component.

These high temperatures indicate that the density structure of the illuminated gas can hardly be uniform with depth. The heating of the uppermost layer by the impinging radiation should result in a density decrease 
in the upper boundary layer. As the result, the effective ionization parameter would be even higher in this upper layer with iron being most likely fully ionized and not contributing to the iron $\mathrm{K} \alpha$ emission and $\mathrm{K}$-shell absorption features observed in Fairall 9 spectrum. Most of the Fe K $\alpha$ line emission and absorption edge would be produced in the coolest, deepest layers of the disc. Nayashin et al. (2000) indicated that the Thomson depth of the top layers is pivotal in determining the fraction of the X-ray fluxes which penetrate to the deeper cooler layers, thereby affecting directly the strength of the Fe line, edge and reflection features. For $\Gamma<2$, these authors found that the equivalent width of the Fe features decreases monotonically with the magnitude of the illuminating flux while the line energy centroid remains at $6.4 \mathrm{keV}$. Remarkably, this conclusion is consistent with our measurement of the Fe $\mathrm{K}$ line equivalent width and $2-10 \mathrm{keV}$ flux being both smaller than those performed by Reynolds (1997) on Fairall 9 spectra obtained in November 1993.

\section{Summary}

We reported an XMM-Newton observation of the Fairall 9 Seyfert 1 galaxy performed in July 2000 with the EPIC cameras and the RGS spectrometers. We conducted the spectral analysis in two steps including first a characterization of the spectrum above $2 \mathrm{keV}$ and then an analysis of the soft $(0.3-2 \mathrm{keV})$ energy range.

Above $2 \mathrm{keV}$, the high signal to noise ratio of the MOS and $\mathrm{p}-\mathrm{n}$ spectra shows an emission line at $6.38 \pm 0.03 \mathrm{keV}$ with a measured equivalent width of about $120 \mathrm{eV}$. An absorption edge is also detected at $7.64_{-0.36}^{+0.21}$ in the source rest frame that has not been reported before. The excellent fit to the measured spectra obtained with reflection models indicates that these imprinted features are likely the result of partial reprocessing of a primary X-ray continuum by optically thick material. The measured energy position of the emission line indicates the presence of iron in a low state of ionization. The position in energy of the absorption edge is consistent with a reflection model from material containing iron in states of ionization lower than FeXVII. The Fe K line observed in the Fairall 9 spectrum is narrow suggesting that the reflection process arises from material relatively far from a putative central black hole. The best fit reflection models point to a photon spectral index of the primary continuum equal to $1.80_{-0.13}^{+0.25}$. This value is higher than the one derived in the $2-5 \mathrm{keV}$ energy band and indicates that the reflected spectrum mask a steeper intrinsic power law slope even at energies lower than $5 \mathrm{keV}$.

We used the determination of the shape of the intrinsic power law above $2 \mathrm{keV}$ to characterize the spectrum of Fairall 9 at low energies. The extrapolation of the primary power law continuum to energies lower than $2 \mathrm{keV}$ indicates the presence of a soft excess component contributing to $14 \pm 6 \%$ of the overall flux in the $0.3-1.0 \mathrm{keV}$ energy range. A redshifted blackbody model with a temperature of $0.17_{-0.07}^{+0.09} \mathrm{keV}$ provides a good description of this component. Confidence contours for temperature versus hydrogen column density support the hypothesis that neutral absorption at low energies is of galactic origin. The ratio of the $\mathrm{p}-\mathrm{n}$ data to the best fit reflection + blackbody model still presents a residual emission feature which deviates significantly from the continuum. A best fit line position is found at $0.60 \mathrm{keV}$ in the source rest frame. However, the absence of line and absorption edges detection in MOS and RGS spectra prevent us to confirm the presence of oxygen in a high state of ionization along the line of sight towards the Fairall 9 nucleus.

Although the geometry of the region emitting the primary X-ray spectrum is not known, the general consensus is that the power law component in the X-ray spectrum of radio-quiet AGNs originates from an extended corona above an accretion disc. Within the frame of such a model, our analysis of Fairall 9 spectra is consistent with the $\mathrm{Fe} \mathrm{K} \alpha$ emission and the absorption edge being produced by Compton reflection of the primary continuum onto an optically thick accretion disk. Our study suggests that the soft-excess emission detected below $2 \mathrm{keV}$ could be interpreted as a bremstrahlung contribution from hot electrons produced by ionization. This interpretation supports models in which X-rays from the primary continuum source heat up the upper layers of the disc thus creating very hot, optically thin plasmas. These plasmas would not only radiate through thermal Bremsstrahlung emission but could upscatter softer photons by inverse Compton effect and reflect part of the primary continuum by Thomson scattering. Hence, the soft excess emission detected in Fairall 9 spectrum would be produced in the upper, ionized layer of the accretion disk while the iron line would mainly originate from deeper, colder regions. The position of the Fe $\mathrm{K}$ absorption edge above $7.1 \mathrm{keV}$ indicates contribution along the line of sight from iron in a range of low ionization states. The profile of the Fe K line suggests that this contribution arises from material relatively far from a putative central black hole for any relativistic effect to be detected.

Acknowledgements. We thank our colleagues from the XMMNewton Science Operation Center for their support in implementing the observations. We are grateful to the anonymous referee for the improvements suggested to an earlier version of the manuscript.

\section{References}

Anders, E., \& Grevesse, N. 1989, Geochim. Cosmochim. Acta, 53, 197

Bambynek, W., Craseman, B., Fink, R. W., et al. 1972, Rev. Mod. Phys., 44, 716

Dickey, J. M., \& Lockman, F. J. 1990, ARA\&A, 28, 215

Fabian, A. C, Rees, M. J., Stella, L., \& White, N. E. 1989, MNRAS, 238, 729

Fairall, A. P. 1977, MNRAS, 180, 391

Ferland, G. J., \& Rees, M. J. 1988, ApJ, 332, 141

Forman, W., Jones, C., Cominsky, L., et al. 1978, ApJS, 38, 357

George, I. M., \& Fabian, A. C. 1991, MNRAS, 249, 352 
Ghisellini, G., Haardt, F., \& Matt, G. 1994, MNRAS, 267, 743 Gondoin, P., Aschenbach, B., Erd, C., et al. 2000, SPIE Proc., 4140, 1

Guainazzi, M., Nicastro, F., Fiore, F., et al. 1998, MNRAS, 301, L1

Guilbert, P. W., \& Rees, M. J. 1988, MNRAS, 233, 475

Haardt, F., \& Matt, G. 1993, MNRAS, 261, 346

den Herder, J. W., Brinkman, A. C., Kahn, S. M., et al. 2001, A\&A, 365, L7

House, L. L. 1969, ApJS, 18, 21

Inoue, H. 1985, in Japan-US Seminar on Galactic and Extragalactic Compact X-Ray Sources, ed. Y. Tanaka, \& W. H. G. Lewin (Tokyo: ISAS), 283

Jansen, F., Lumb, D., Altieri, B., et al. 2001, A\&A, 365, L1

Kellog, et al. 1975, ApJ, 199, 299

Krolik, J. H., Mc Kee, C. F., \& Tarter, C. B. 1981, ApJ, 249, 422

Krolik, J. H., Madau, P., \& Zycki, P. 1994, ApJ, 420, L57

Laor, A. 1991, ApJ, 376, 90

McHardy, I, Lawrence, A., Pye, J. P., \& Pounds, K. A. 1981, MNRAS, 197, 893

Magdziar, P., \& Zdziarski, A. A. 1995, MNRAS, 273, 837

Makishima, K, 1986, in The Physics of Accretion onto Compact Objects, ed. K. O. Mason, M. G. Watson, \& N. E. White (Springer Verlag, Berlin), 250

Martocchia, A., \& Matt, G. 1996, MNRAS, 282, L53

Matt, G., Perola, G. C., \& Piro, L. 1991, A\&A, 247, 25

Matt, G., Perola, G. C., Piro, L., \& Stella, L. 1992a, A\&A, 257, 63

Matt, G., \& Perola, G. C. 1992b, MNRAS, 259, 433

Matt, G., Fabian, A. C., \& Ross, R. R. 1994, MNRAS, 262, 179

Morini, M., Scarsi, L., Molteni, D., et al. 1986, ApJ, 307, 486

Morita, S., \& Fujita, J. 1983, J. Phys. Soc. Jpn, 52, 1957

Morrison, R., \& McCammon, D. 1983, ApJ, 270, 119

Mushotzky, R. F., Fabian, A. C., Iwasawa, K., et al. 1995, MNRAS, 272, L9

Nandra, K., George, I. M., Mushotzky, R. F., et al. 1997, ApJ, 477, 602

Narayan, R., \& Yi I. 1995, ApJ, 444, 231

Nayakshin, S., Kazanas, D., \& Kallman, T. R. 2000, ApJ, 537, 833
Page, M. J., Mason, K. O., Carrera, F. J., et al. 2001, A\&A, 365, L152

Petre, R., Mushotzki, R. F., Krolik, J. H., \& Holt, S. S. 1984, ApJ, 280, 499

Piccinoti, G. Mushotzki, R. F., Krolik, J. H., et al. 1982, ApJ, 253, 485

Piro, L., Balucinska-Church, M., Fink, H., et al. 1997, A\&A, 319, 74

Pounds, K. A., Nandra, K., Stewart, G. C., et al. 1990, Nature, 344,132

Pounds, K. A., Nandra, K., Fink, H. H., \& Makino, F. 1994, MNRAS, 267, 193

Recondo-Gonzales, M. C., Wamsteker, W., Clavel, J., et al. 1997, A\&AS, 121, 461

Rees, M. J. 1984, ARA\&A, 22, 471

Reeves, J. N., Turner, M. J. L., Bennie, P. J., et al. 2001a, A\&A, 365, L116

Reeves, J. N., Turner, M. J. L., Pounds, K. A., et al. 2001b, A\&A, 365, L134

Reynolds, C. S. 1997, MNRAS, 286, 513

Reynolds, C. S., \& Begelman, M. C. 1997, ApJ, 488, 109

Reynolds, C. S., \& Fabian, A. C. 1997, MNRAS, 290, L1

Ross, R. R., \& Fabian, A. C. 1993, MNRAS, 261, 74

Ross, R. R., Fabian, A. C., \& Young, A. J. 1999, MNRAS, 306, 461

Stella, L. 1990, Nature, 344, 747

Tanaka, Y., Nandra, K., \& Fabian, A. C. 1995, Nature, 375, 659

Turner, T. J., \& Pounds, K. A. 1989, MNRAS, 240, 833

Turner, T. J., Abbey, A., Arnaud, M., et al. 2001, A\&A, 365, L27

Walter, R., \& Fink, H. H. 1993, A\&A, 274, 105

Ward, M. J., \& Wilson, A. S. 1978, ApJ, 223, 788

Weaver, K. A., Nousek, J., Yaqoob, T., et al. 1996, ApJ, 458, 160

Wood, K. S., et al. 1984, ApJS, 56, 507

Yaqoob, T., George, I. M., Nandra, K., et al. 2001, ApJ, 546, 759

Zycki, P. T., Krolik, J. H., Zdiarski, A. A., \& Kallman, T. R. 1994, ApJ, 437, 597 